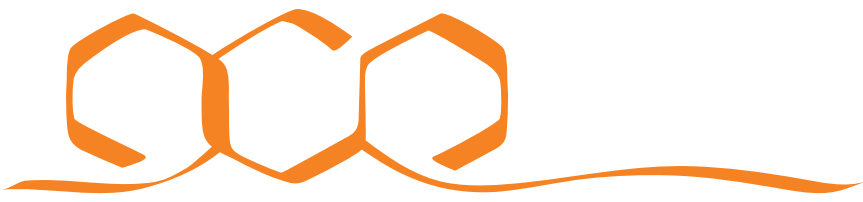 \\ COMMUNICATIONS CHEMISTRY
}

ARTICLE

https://doi.org/10.1038/s42004-020-00366-1 OPEN

\section{Chiral lanthanide lumino-glass for a circularly polarized light security device}

Yuichi Kitagawa ${ }^{1,2,5}{ }^{\star}$, Satoshi Wada ${ }^{1,5}$, M. D. Jahidul Islam² ${ }^{2}$ Kenichiro Saita ${ }^{3}{ }^{3}$, Masayuki Gon ${ }^{4}$, Koji Fushimi ${ }^{1}$, Kazuo Tanaka (1] ${ }^{4}$, Satoshi Maeda ${ }^{2,3} \&$ Yasuchika Hasegawa ${ }^{1,4 凶}$

Artificial light plays an essential role in information technologies such as optical telecommunications, data storage, security features, and the display of information. Here, we show a chiral lanthanide lumino-glass with extra-large circularly polarized luminescence (CPL) for advanced photonic security device applications. The chiral lanthanide glass is composed of a europium complex with the chiral (+)-3-(trifluoroacetyl)camphor ligand and the achiral glass promoter tris(2,6-dimethoxyphenyl)phosphine oxide ligand. The glass phase transition behavior of the Eu(III) complex is characterized using differential scanning calorimetry. The transparent amorphous glass shows CPL with extra-large dissymmetry factor of $g_{\mathrm{CPL}}=1.2$. The brightness of the lumino-glass is one thousand times larger than that of Eu(III) luminophores embedded in polymer films of the same thickness at a Eu(III) concentration of $1 \mathrm{mM}$. The application of the chiral lanthanide lumino-glass in an advanced security paint is demonstrated.

\footnotetext{
${ }^{1}$ Faculty of Engineering, Hokkaido University, N13 W8, Kita-ku, Sapporo, Hokkaido 060-8628, Japan. ${ }^{2}$ Institute for Chemical Reaction Design and Discovery (WPI-ICReDD), Hokkaido University, N21 W10, Sapporo, Hokkaido 001-0021, Japan. ${ }^{3}$ Faculty of Science, Department of Chemistry, Hokkaido University, Sapporo 060-0810, Japan. ${ }^{4}$ Graduate School of Engineering, Kyoto University, Katsura, Nishikyo-ku, Kyoto 615-8510, Japan. ${ }^{5}$ These authors contributed

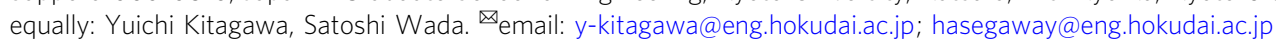


A rtificial light plays an essential role in information technologies such as optical telecommunications, data storage, security features, and the display of information ${ }^{1}$. Organicbased luminophores that allow precise control of information have been extensively studied for the development of light-based information technologies ${ }^{2,3}$. This precise control originates from the molecular quantum design of organic luminophores ${ }^{4-6}$. The combination of the molecules with quantum design and flexible amorphous materials is expected to result in advancements in photo-science and -technology for industrial applications such as flexible organic light-emitting diodes (OLED) $)^{7-9}$.

In this work, we focus on an optical information technology based on chiral luminescent molecules. Recently, circularly polarized luminescence (CPL) from chiral organic molecules has attracted considerable attention because of its applications in sophisticated photo-information technologies, such as security tags, lasers, data storage, and organic electroluminescent (EL) devices for three-dimensional displays ${ }^{10-16}$. The CPL phenomena are characterized by the differential emission of right- and lefthanded circularly polarized light. The magnitude of CPL is given by the following equation 17,18 :

$$
g_{\mathrm{CPL}}=2 \frac{I_{\mathrm{L}}-I_{\mathrm{R}}}{I_{\mathrm{L}}+I_{\mathrm{R}}}
$$

where $I_{\mathrm{L}}$ and $I_{\mathrm{R}}$ are the emission intensities of the left- and righthanded CPL, respectively.

In particular, lanthanide complexes containing chiral organic ligands exhibit dissymmetry factors $\left(g_{\mathrm{CPL}}\right)$ approximately a thousand times larger than those of chiral organic molecules ${ }^{10-13,19-22}$. Parker prepared anion sensors using the CPL signals of mononuclear $\mathrm{Eu}(\mathrm{III})$ and $\mathrm{Tb}(\mathrm{III})$ complexes with chiral ligands incorporating an azaxanthone sensitizer ${ }^{23}$. Muller and colleagues ${ }^{24}$ observed an exceptionally large CPL in an Eu(III)-Cs(I) system with characteristic chiral $\beta$-diketonate ligands containing a camphor framework (tetrakis(3-heptafluorobutylryl- $(+)$-camphorato) $\left(g_{\mathrm{CPL}}=-1.38\right)^{24}$, although the emission quantum yield was extremely low $\left(\Phi_{\text {tot }}<1.0 \%\right)^{25}$. Recently, we found that phosphine oxide ligands improve the quantum emission yield by controlling the energy quenching state for a chiral $\mathrm{Eu}(\mathrm{III})$ complex with camphor $\left(\Phi_{\text {tot }}>10 \%\right)^{26}$. The use of well-designed phosphine oxide ligands is a key strategy for the construction of chiral $\mathrm{Eu}$ (III) complexes with large $g_{\mathrm{CPL}}$ and $\Phi_{\text {tot }}$ values for advanced CPL luminophore applications.

Herein, we prepare a bulky phosphine oxide ligand with six methoxy groups, tmpo (tris(2,6-dimethoxyphenyl)phosphine oxide, Fig. 1a), for the construction of complexes with large $g_{\mathrm{CPL}}$ and $\Phi_{\text {tot }}$ values. The bulky ligand coordinates weakly to the Eu(III) ion; such weak coordination has been reported to be related to the enhancement of $\mathrm{CPL}^{22}$. The tmpo ligand is also expected to promote glass formation due to the multiple coordination sites provided by its phosphine oxide $(\mathrm{P}=\mathrm{O})^{26}$ and methoxy groups $(\mathrm{O}-\mathrm{Me})^{27}$, which is beneficial for the fabrication of transparent materials. The ligand $( \pm)-3$-(trifluoroacetyl)camphor $( \pm t f c)$ is selected to achieve a chiral ligand arrangement around the Eu center (Fig. 1b). The chiral Eu(III) complexes with phosphine oxide ligands are prepared by casting $\mathrm{Eu}( \pm \mathrm{tfc})_{3}\left(\mathrm{H}_{2} \mathrm{O}\right)_{2}$ and tmpo ligands on a substrate. The glass phase transition behavior of the $\mathrm{Eu}(\mathrm{III})$ complex $(\mathrm{Eu}( \pm \mathrm{tfc})$-tmpo) is characterized using differential scanning calorimetry. The resulting chiral Eu(III) lumino-glass exhibits extraordinary CPL intensity $\left(g_{\mathrm{CPL}}=1.2\right)$ and a relatively high emission quantum yield $\left(\Phi_{\text {tot }}=13 \%\right)$. Chiral Eu(III) complexes with other phosphine oxide ligands (Fig. 1c, L1: Tris(2methoxyphenyl)phosphine oxide, L2: Tris(3-methoxyphenyl) phosphine oxide, L3: tris(4-methoxyphenyl)phosphine oxide, L4: tris(4-methylphenyl)phosphine oxide, L5: tris(2,4,6-trimethylphenyl)phosphine oxide, Supplementary Methods) are also prepared (a)

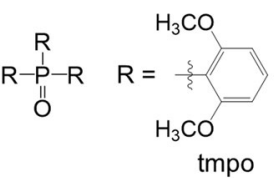

(b)

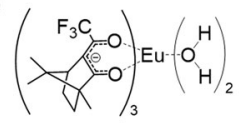

(c)

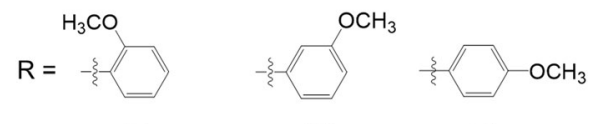

$\mathrm{L} 1$

L2

L3

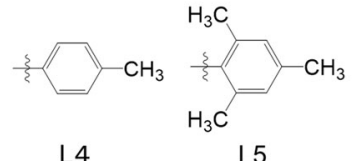

L4

L5

Fig. 1 Molecular structures. Chemical structures of tmpo ligand (a), Eu $(+\mathrm{tfc})_{3}\left(\mathrm{H}_{2} \mathrm{O}\right)_{2}(\mathbf{b}), \mathrm{L} 1, \mathrm{~L} 2, \mathrm{~L} 3, \mathrm{~L} 4$, and $\mathrm{L} 5$ ligands (c). (a) UV-off

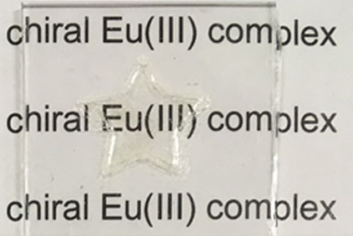

(b) UV-on

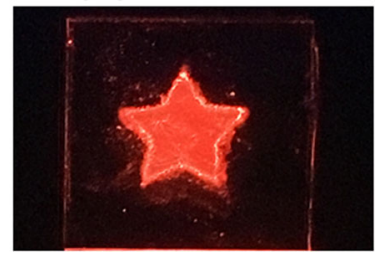

Fig. 2 Photographs of Eu(III) lumino-glass. Photographs of Eu(III) luminoglass (Eu(+tfc)-tmpo(2). UV-off (a), UV-on (b, $\left.\lambda_{\mathrm{ex}}=365 \mathrm{~nm}\right)$ ).

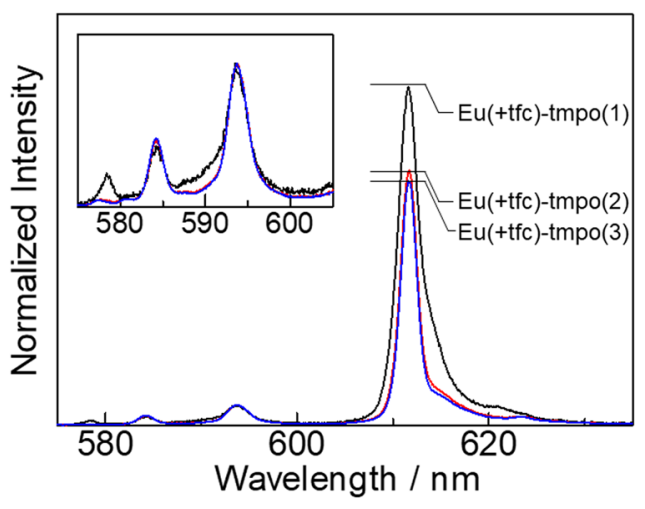

Fig. 3 Emission spectra. Emission spectra of $E u(+t f c)-t m p o(1)$ (black line), $\mathrm{Eu}(+\mathrm{tfc})-\mathrm{tmpo}(2)$ (red line), and $\mathrm{Eu}(+\mathrm{tfc})-\mathrm{tmpo}$ (3) (blue line).

for comparison of their photophysical properties. Based on spectroscopic analyses, we determine that the extra-large $g_{\mathrm{CPL}}$ of Eu $( \pm \mathrm{tfc})$-tmpo originates from its characteristic symmetric coordination structure with a strong crystal field. Using the chiral Eu(III) lumino-glass, the first application of CPL paint as a security ink (to display a sun or moon shape) are demonstrated. These glasstype chiral $\mathrm{Eu}(\mathrm{III})$ complexes are promising candidates for future devices with polarized light information.

\section{Results and discussion}

Luminescence intensity of Eu(III) lumino-glass. Emission properties of $\mathrm{Eu}$ (III) lumino-glass (Fig. 2) were evaluated using emission spectra and time-resolved emission measurement. The emission spectra of the $\mathrm{Eu}(+\mathrm{tfc})-\operatorname{tmpo}(n)$ films $(n=1,2,3, n$ : number of equivalents relative to $\left.\mathrm{Eu}(+\mathrm{tfc})_{3}\right)$ are shown in Fig. 3 (Supplementary Note 1 and Supplementary Figs. 1-3). The 
Table 1 Photophysical properties of Eu(III) lumino-glass.

\begin{tabular}{|c|c|c|c|c|}
\hline & $\tau_{1}(\mathrm{~ms})^{\mathrm{a}}$ & $\tau_{2}(\mathrm{~ms})^{\mathrm{a}}$ & $\tau_{3}(\mathrm{~ms})^{\mathrm{a}}$ & $\Phi_{\text {tot }}(\%)^{b}$ \\
\hline $\mathrm{Eu}(+\mathrm{tfc})-\mathrm{tmpo}(1)$ & $0.10(11 \%)$ & $0.28(44 \%)$ & $0.70(45 \%)$ & 2.6 \\
\hline $\mathrm{Eu}(+\mathrm{tfc})-\mathrm{tmpo}(2)$ & $0.14(6 \%)$ & $0.37(34 \%)$ & $0.74(60 \%)$ & 13 \\
\hline $\mathrm{Eu}(+\mathrm{tfc})-\mathrm{tmpo}(3)$ & $0.16(5 \%)$ & $0.41(34 \%)$ & $0.76(61 \%)$ & 13 \\
\hline
\end{tabular}

spectra were normalized using the integrated intensity of the magnetic dipole transition (MD: ${ }^{5} \mathrm{D}_{0} \rightarrow{ }^{7} \mathrm{~F}_{1}$ ). Two peaks were observed at 584 and $594 \mathrm{~nm}$ for the MD transition (Fig. 3, inset). The strong emission bands near $612 \mathrm{~nm}$ were attributed to the electric dipole transition (ED: ${ }^{5} \mathrm{D}_{0} \rightarrow{ }^{7} \mathrm{~F}_{2}$ ). The emission band of $\mathrm{Eu}(+\mathrm{tfc})-\mathrm{tmpo}(1)$ was different from those of $\mathrm{Eu}(+\mathrm{tfc})-\mathrm{tmpo}(2)$ and $\mathrm{Eu}(+\mathrm{tfc})-\mathrm{tmpo}(3)$. The luminescence intensity ratio of the $\mathrm{ED} / \mathrm{MD}$ transition $\left(\mathrm{A}_{\mathrm{ED}} / \mathrm{A}_{\mathrm{MD}}\right)$ decreased with increasing $n$ (i.e., increasing number of tmpo molecules), indicating that the $\mathrm{Eu}(\mathrm{III})$ complex with tmpo ligands forms a symmetric coordination geometry ${ }^{28}$. The crystal field splitting in the $\mathrm{MD}$ transition was found to be $274 \mathrm{~cm}^{-1}$. Small $\mathrm{A}_{\mathrm{ED}} / \mathrm{A}_{\mathrm{MD}}$ and large crystal field splitting values were also observed in a comparative study with other Eu(III) complexes (Supplementary Note 2-3, Supplementary Figs. 4-7, and Supplementary Table 1). These results indicated that a symmetric coordination structure with a strong crystal field was formed by the tmpo ligands, which was attributed to the large steric hindrance and multiple interaction of tmpo ligands.

To obtain information regarding the coordination structure around the luminescent $\mathrm{Eu}(\mathrm{III})$ center, the time-resolved emission profiles of the complexes were measured. The emission lifetimes were estimated using triple exponential decays (Table 1), and were consistent with the existence of several metastable structures. The relative lifetime of the $\tau_{3}$ component increased with the addition of more equivalents of tmpo. The emission decay phenomenon of $\mathrm{Eu}(+\mathrm{tfc})$-tmpo(2) was similar to that of $\mathrm{Eu}$ $(+\mathrm{tfc})$-tmpo(3). The emission quantum efficiencies excited by the tfc ligands $\left(\Phi_{\text {tot }}\right)$ of the films are also shown in Table 1. The $\Phi_{\text {tot }}$ values also increased with the number of equivalents of tmpo added. The emission quantum yield of $\mathrm{Eu}(+\mathrm{tfc})-\mathrm{tmpo}(2)\left(\Phi_{\mathrm{tot}}=\right.$ $13 \%)$ was much larger than that of previously reported $\mathrm{Eu}(\mathrm{III})$ $\mathrm{Cs}(\mathrm{I})$ systems $\left(\Phi_{\mathrm{tot}}<1 \%\right)$ with a large $g_{\mathrm{CPL}}$ value $(-1.38)$ in solution ${ }^{24,25}$.

CPL properties of $E \mathbf{u}( \pm t f c)$ with tmpo ligands. The CPL spectra of $\mathrm{Eu}( \pm \mathrm{tfc}$ )-tmpo(2) are shown in Fig. 4 (Supplementary Note 4-6, Supplementary Figs. 8-15, and Supplementary Table 2). Large CPL signals were observed for the $\mathrm{MD}$ transition at $594 \mathrm{~nm}(\mathrm{Eu}(+\mathrm{tfc})-$ tmpo(2): $\left.g_{\mathrm{CPL}}=-1.2, \mathrm{Eu}(-\mathrm{tfc})-\mathrm{tmpo}(2): g_{\mathrm{CPL}}=1.2\right)$ and the $\mathrm{ED}$ transition at $612 \mathrm{~nm}\left(\mathrm{Eu}(+\mathrm{tfc})\right.$-tmpo(2): $g_{\mathrm{CPL}}=0.15, \mathrm{Eu}(-\mathrm{tfc})$-tmpo (2): $\left.g_{\mathrm{CPL}}=-0.15\right)$. The $g_{\mathrm{CPL}}$ value in the MD transition is almost same as that of $\mathrm{Eu}(+\mathrm{tfc})-\mathrm{tmpo}(1)\left(g_{\mathrm{CPL}}=-1.0\right)$ and $\mathrm{Eu}(+\mathrm{tfc})-$ tmpo(3) $\left(g_{\mathrm{CPL}}=-1.2\right.$; Supplementary Note 7 and Supplementary Fig. 16). The CPL spectrum of $\mathrm{Eu}(+\mathrm{tfc})$ with two equivalents of tmpo in solution also exhibited a large CPL signal $\left(g_{\mathrm{CPL}}=-1.0\right)$ (Supplementary Notes 8 and 9, Supplementary Figs. 17-19, Supplementary Table 3, and Supplementary Data 1-3). The $g_{\mathrm{CPL}}$ values at $594 \mathrm{~nm}$ (film: $g_{\mathrm{CPL}}=-1.2$, solution: $g_{\mathrm{CPL}}=-1.0$ ) were as large as that previously reported $\left(g_{\mathrm{CPL}}=-1.38\right)$ for $\mathrm{Eu}(\mathrm{III})-\mathrm{Cs}(\mathrm{I})$ systems $^{24,25}$. These values were different from the small $g_{\mathrm{CPL}}$ value of a mononuclear Eu (III) complex with tightly coordinated phosphine oxide ligands $\left(\mathrm{Eu}(+\mathrm{tfc})_{3}(\mathrm{tppo})_{2}\right.$, tppo: triphenylphosphine oxide, $\left.g_{\mathrm{CPL}}=0.09\right)$ and a polynuclear $\mathrm{Eu}(\mathrm{III})$ complex $\left(\left[\mathrm{Eu}(+\mathrm{tfc})_{3} \mathrm{dpbp}\right]_{n}\right.$, dpbp: 4,4-bis

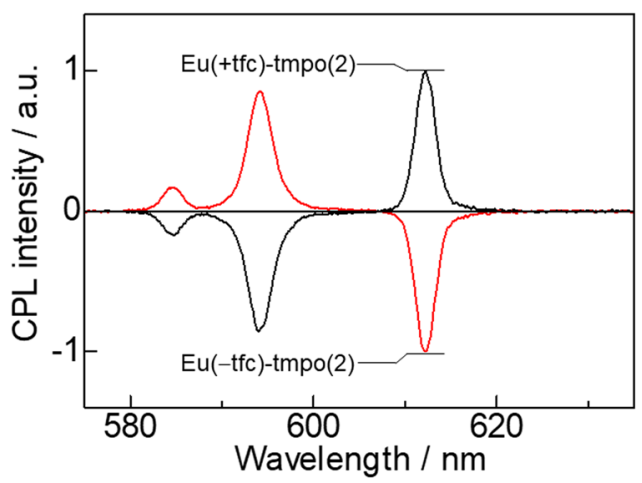

Fig. 4 CPL spectra. CPL spectra of $\mathrm{Eu}(+\mathrm{tfc})$-tmpo(2) (black line) and $\mathrm{Eu}$ (-tfc)-tmpo(2) (red line).

(diphenylphosphoryl)biphenyl, $\left.g_{\mathrm{CPL}}=0.17\right)^{26}$. The $g_{\mathrm{CPL}}$ value of $\mathrm{Eu}$ $(+\mathrm{tfc})$-tmpo(2) was also higher than those of the other $\mathrm{Eu}(\mathrm{III})$ lumino-glasses in a comparative study (Supplementary Fig. 7 and Supplementary Table 1). In the comparative compounds, the $\mathrm{Eu}$ $(+\mathrm{tfc})-\mathrm{L} 1(2)$ showed a relatively large $g_{\mathrm{CPL}}$ signal. We attributed the large $g_{\mathrm{CPL}}$ value of the $\mathrm{Eu}(\mathrm{III})$ lumino-glass to the large J-mixing (J: total angular momentum $)^{22}$ induced by the symmetric coordination geometry with a strong crystal field. In addition, the methoxy group in the ortho-position in the phosphine oxide ligands play an important role in the formation of the characteristic coordination field.

Demonstration of a CPL application. We also demonstrated a CPL paint application using the amorphous $\mathrm{Eu}(\mathrm{III})$ lumino-glass. The prepared samples are shown in Fig. 5a. Transparent glasses of $\mathrm{Eu}(+\mathrm{tfc})-\mathrm{tmpo}(2)$ and its enantiomer $(\mathrm{Eu}(-\mathrm{tfc})-\mathrm{tmpo}(2))$ were prepared on a substrate using a casting method $(\mathrm{Eu}(+\mathrm{tfc})$-tmpo (2): sun symbol; $\mathrm{Eu}(-\mathrm{tfc})-\mathrm{tmpo}(2)$ : moon symbol). The experimental setup for visualizing the CPL image is depicted in Fig. 5b. In the setup, the emission of the lumino-glasses is detected using a camera with a linear polarizer, a rotatable $\lambda / 4$ plate, and a bandpass filter $(594 \mathrm{~nm})$. The left- or right-handed polarized light is detected by rotating the $\lambda / 4$ plate $90^{\circ}$ clockwise or anticlockwise using an angle controller. The photographs of the emission of the samples using total light (without the $\lambda / 4$ plate), left-handed, and right-handed circularly polarized light are shown in Fig. $5 \mathrm{c}$, d, and e, respectively. The total emission from the $\mathrm{Eu}(+)$ and $\mathrm{Eu}(-)$ lumino-glass is observed in photograph (Fig. 5c). Under clockwise and anticlockwise rotation, the sun and moon shapes of the $\mathrm{Eu}(+)$ and $\mathrm{Eu}(-)$ lumino-glasses were observed, respectively (photographs Fig. 5d, e). This is the first demonstration of nakedeye detection of a security paint based on the CPL phenomenon using amorphous $\mathrm{Eu}(\mathrm{III})$ lumino-glasses.

To the best of our knowledge, the first lanthanide lumino-glass with an extra-large CPL and a relatively high emission quantum yield was reported. We revealed that symmetric coordination with a strong crystal field is a key factor for the enhancement of 
(a) Film samples

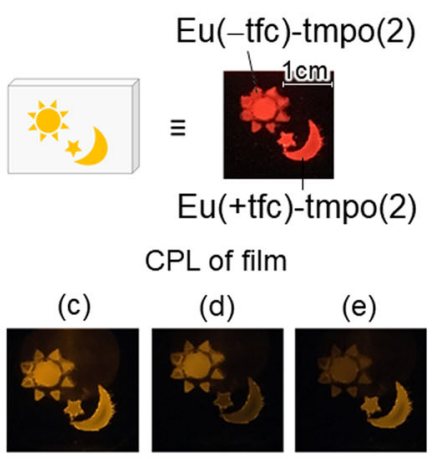

(b) CPL detection setup

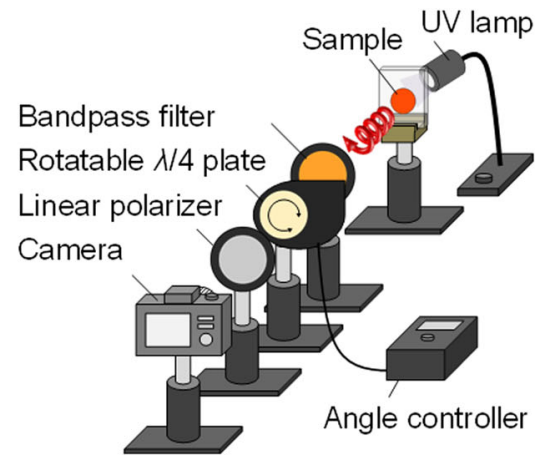

Fig. 5 CPL detection. a Photographs and images of the films of Eu( $+\mathrm{tfc})$-tmpo(2) and Eu(-tfc)-tmpo(2) prepared on glasses under UV-light $\left(\lambda_{\mathrm{ex}}=365\right.$ $\mathrm{nm})$. b The simple setup for CPL detection. Photographs of the emission $\left(\lambda_{\mathrm{em}}=594 \mathrm{~nm}\right)$ related to $\mathbf{c}$ left and right, $\mathbf{d}$ left, or $\mathbf{e}$ right circularly polarized light from the samples.

$g_{\mathrm{CPL}}$. We also successfully demonstrated a security paint based on the CPL phenomenon using Eu(III) lumino-glass for the first time. Our results provide new insights into the design of $\mathrm{Eu}(\mathrm{III})$ complexes with excellent CPL performance and high emission intensity, which should lead to new applications of CPL materials as security inks.

\section{Methods}

Materials. Europium(III) acetate $n$-hydrate, acetone- $\mathrm{d}_{6}(99.9 \%)$ and $28 \%$ ammonia solution were purchased from Wako Pure Chemical Industries Ltd. Tris(2,4,6trimethylphenyl)phosphine, $(+)$-3-(trifluoroacetyl)camphor and (-)-3-(trifluoroacetyl)camphor were purchased from Sigma-Aldrich Co. Tris(2,6-dimethoxyphenyl)phosphine, tris(4-methoxyphenyl)phosphine, and tris(4methylphenyl)phosphine were purchased from Tokyo Chemical Industry Co., Ltd. All other chemicals and solvents were of reagent grade and were used without further purification.

Apparatus. Electrospray ionization mass spectra (ESI-MS) were measured by using a Thermo Scientific Exactive instrument. Elemental analyses were performed on an Exeter Analytical CE440. Emission spectra and emission lifetimes were measured using a Horiba/Jobin-Yvon FluoroLog-3 spectrofluorometer. CPL spectra were measured using a JASCO CPL-300 spectrofluoropolarimeter. DSC measurements were recorded on a SII DSC 7020 heat flux meter. Proton nuclear magnetic resonance $\left({ }^{1} \mathrm{H}\right.$ NMR $)$ spectra were recorded on an auto-NMR JEOL ECS $400 \mathrm{MHz}$.

Synthesis of Tris(2,6-dimethoxyphenyl)phosphine oxide. Tris(2,6-dimethoxyphenyl)phosphine $(8.84 \mathrm{~g}, 0.02 \mathrm{~mol})$ was dissolved in dichloromethane $(30 \mathrm{~mL})$ in a 100 -mL flask, which was cooled in an ice bath. $\mathrm{H}_{2} \mathrm{O}_{2}$ solution $(5 \mathrm{~mL})$ was added slowly to the solution. The mixture was stirred for $3 \mathrm{~h}$. The product was extracted with dichloromethane and saturated $\mathrm{NaCl}$ aqueous solution. The organic layer was dried with anhydrous $\mathrm{MgSO}_{4}$, and the solvent was evaporated. The obtained oil was precipitated with acetone and hexane. The precipitate was filtered and washed with acetone to afford white powder.

Yield: $3.95 \mathrm{~g}(41 \%) . \delta / \mathrm{ppm}=7.23(\mathrm{t}, 3 \mathrm{H}, J=8 \mathrm{~Hz}, \mathrm{Ar}), 6.48(\mathrm{dd}, 6 \mathrm{H}, J=8$ and $4.8 \mathrm{~Hz}, \mathrm{Ar}), 3.51\left(\mathrm{~s}, 18 \mathrm{H}, \mathrm{CH}_{3}\right)$. ESI-MS $(\mathrm{m} / \mathrm{z}):[\mathrm{M}+\mathrm{H}]^{+}$calculated for $\mathrm{C}_{24} \mathrm{H}_{28} \mathrm{O}_{7} \mathrm{P}$, 459.15; found, 459.16. Elemental analysis: Calcd for $\mathrm{C}_{24} \mathrm{H}_{27} \mathrm{O}_{7} \mathrm{P}: \mathrm{C}, 62.88 \%, \mathrm{H}$, $5.94 \%$. Found: C, $62.55 \%, \mathrm{H}, 5.88 \%$.

Synthesis of Tris(3-trifluoroacetyl-( + )-camphorato)europium(III). Europium (III) acetate $n$-hydrate $(0.36 \mathrm{~g})$ was dissolved in distilled water $(150 \mathrm{~mL}) .(+)-3$ triflouroacetyl camphor $(+\mathrm{tfc}, 0.50 \mathrm{~g}, 2.0 \mathrm{mmol})$ in methanol $(20 \mathrm{~mL})$ was added to the solution. A few drops of $28 \%$ ammonia solution were added and the mixture was stirred for $3 \mathrm{~h}$ at room temperature. The obtained powder was washed with distilled water to afford yellow powder.

Yield: $0.42 \mathrm{~g}(68 \%)$. Elemental analysis: Calcd for $\mathrm{C}_{36} \mathrm{H}_{46} \mathrm{EuF}_{9} \mathrm{O}_{8}$ : $\mathrm{C}, 46.51 \%, \mathrm{H}$, $4.99 \%$. Found: C, $46.45 \%$, H, $4.91 \%$.

Synthesis of Tris(3-trifluoroacetyl-(-)-camphorato)europium(III). [Eu $\left.(-\mathrm{tfc})_{3}\left(\mathrm{H}_{2} \mathrm{O}\right)_{2}\right]$ was prepared using the same method for $\left[\mathrm{Eu}(+\mathrm{tfc})_{3}\left(\mathrm{H}_{2} \mathrm{O}\right)_{2}\right]$, starting from (-)-3-triflouroacetyl camphor, yielding yellow powder.

Preparation of Eu(III) lumino-glass. $\mathrm{Eu}( \pm \mathrm{tfc})_{3}\left(\mathrm{H}_{2} \mathrm{O}\right)_{2}(3 \mathrm{mg}, 0.003 \mathrm{mmol})$ and $n$ equivalents of tmpo (1.5 mg, $3.0 \mathrm{mg}$, or $4.5 \mathrm{mg}$ for $n=1,2$, or 3 , respectively) were dissolved in dichloromethane $(0.1 \mathrm{~mL})$ (Supplementary Note 1 and Supplementary Fig. 1). The solution was casted onto a glass substrate and allowed to slowly evaporate, yielding a transparent film of the $\mathrm{Eu}(+\mathrm{tfc})-\operatorname{tmpo}(n)(n=1,2,3, n$ : number of equivalents relative to $\left.\mathrm{Eu}(+\mathrm{tfc})_{3}\right)$. The obtained film was characterized by ESI-MS, XRD (Supplementary Fig. 2), and DSC (Supplementary Fig. 3). ESI mass spectrometry revealed the existence of the Eu(III) complex [Eu $\left.(+\mathrm{tfc})_{2}(\mathrm{tmpo})_{2}\right]^{+}$(cacld: 1563.41 , found: 1563.38). The XRD signals of the transparent film were broad, similar to those of typical amorphous organic molecules. In the DSC curve, a characteristic endothermic peak with shoulders indicating a glass transition was observed at $\sim 50{ }^{\circ} \mathrm{C}$ (Supplementary Fig. 3). The glass-typed Eu(III) complexes showed bright red luminescence ("Eu(III) lumino-glass", ex. $\mathrm{Eu}(+\mathrm{tfc})-$ tmpo(2), Fig. 2).

\section{Data availability}

The authors declare that the data supporting the findings of this study are available within the paper and its supplementary information.

Received: 4 May 2020; Accepted: 31 July 2020; Published online: 25 August 2020

\section{References}

1. Miolo, G., Stair, J. L. \& Zloh, M. Light In Forensic Science: Issues And Applications (RSC, 2018).

2. Xu, S., Chen, R., Zheng, C. \& Huang, W. Excited state modulation for organic afterglow: materials and applications. Adv. Mater. 28, 9920 (2016).

3. Xu, S., Duan, Y. \& Liu, B. Precise molecular design for high-performance luminogens with aggregation-induced emission. Adv. Mater. 32, 1903530 (2019).

4. Liang, X., Tu, Z.-L. \& Zheng, Y.-X. Thermally activated delayed fluorescence materials: Towards realization of high efficiency through strategic small molecular design. Chem. Eur. J. 25, 5623 (2019).

5. Li, L.-K. et al. Strategies towards rational design of gold(III) complexes for high-performance organic light-emitting devices. Nat. Photon. 13, 185 (2019).

6. Ma, H., Peng, Q., An, Z., Huang, W. \& Shuai, Z. Efficient and long-lived room-temperature organic phosphorescence: Theoretical descriptors for molecular designs. J. Am. Chem. Soc. 141, 1010 (2019).

7. Wu, X., Jin, M., Xie, J., Malval, J.-P. \& Wan, D. Molecular engineering of UV/ Vis light-emitting diode (LED)-sensitive donor- $\pi$-acceptor-type sulfonium salt photoacid generators: Design, synthesis, and study of photochemical and photophysical properties. Chem. Eur. J. 23, 15783 (2017).

8. Noda, T., Ogawa, H. \& Shirota, Y. A blue-emitting organic electroluminescent device using a novel emitting amorphous molecular material, 5,5'-Bis (dimesitylboryl)-2,2'-bithiophene. Adv. Mater. 11, 283 (1999).

9. Hirata, S. et al. Efficient persistent room temperature phosphorescence in organic amorphous materials under ambient conditions. Adv. Funct. Mater. 23, 3386 (2013).

10. Zinna, F. et al. Design of lanthanide-based OLEDs with remarkable circularly polarized electroluminescence. Adv. Funct. Mater. 27, 1603719 (2017).

11. Muller, G. Luminescent chiral lanthanide(III) complexes as potential molecular probes. Dalton Trans. 44, 9692 (2009).

12. Carr, R., Evans, N. H. \& Parker, D. Lanthanide complexes as chiral probes exploiting circularly polarized luminescence. Chem. Soc. Rev. 41, 7673 (2012)

13. Zinna, F. \& Di Bari, L. Lanthanide circularly polarized luminescence: bases and applications. Chirality 27, 1 (2015). 
14. Yang, X., Han, J., Wang, Y. \& Duan, P. Photon-upconverting chiral liquid crystal: significantly amplified upconverted circularly polarized luminescence. Chem. Sci. 10, 172 (2019).

15. Song, F. et al. Highly efficient circularly polarized electroluminescence from aggregation-induced emission luminogens with amplified chirality and delayed fluorescence. Adv. Funct. Mater. 28, 1800051 (2018).

16. Sánchez-Carnerero, E. M. et al. Circularly polarized luminescence from simple organic molecules. Chem. Eur. J. 21, 13488 (2015).

17. Mason, S. F. Molecular Optical Activity And The Chiral Discriminations (Cambridge University Press, 1982).

18. Riehl, J. P. \& Richardson, F. S. Circularly polarized luminescence spectroscopy. Chem. Rev. 86, 1 (1986).

19. Brittain, H. G. \& Richardson, F. S. Circularly polarized emission studies on the chiral nuclear magnetic resonance lanthanide shift reagent tris(3trifluoroacetyl-d-camphorato)europium(III). J. Am. Chem. Soc. 98, 5858 (1976).

20. Brittain, H. G., Richardson, F. S. \& Martin, R. B. Terbium(III) emission as a probe of calcium(II) binding sites in proteins. J. Am. Chem. Soc. 98, 8255 (1976).

21. Spaulding, L. \& Brittain, H. G. Complexation of amino-acids by terbium(III) ethylenediaminetetraacetate. Inorg. Chem. 24, 3692 (1985).

22. Wada, S. et al. Electronic chirality inversion of lanthanide complex induced by achiral molecules. Sci. Rep. 8, 16395 (2018).

23. Smith, D. G., Pal, R. \& Parker, D. Measuring equilibrium bicarbonate concentrations directly in cellular mitochondria and in human serum using europium/terbium emission intensity ratios. Chem. Eur. J. 18, 11604 (2012).

24. Lunkley, J. L., Shirotani, D., Yamanari, K., Kaizaki, S. \& Muller, G. Chiroptical spectra of a series of tetrakis $(+)$-3-heptafluorobutylrylcamphorato) lanthanide(III) with an encapsulated alkali metal ion: circularly polarized luminescence and absolute chiral structures for the Eu(III) and Sm(III) complexes. Inorg. Chem. 50, 12724 (2011).

25. Kumar, J., Marydasan, B., Nakashima, T., Kawai, T. \& Yuasa, J. Chiral supramolecular polymerization leading to eye differentiable circular polarization in luminescence. Chem. Commun. 52, 9885 (2016).

26. Hasegawa, Y. et al. Spiral Eu(III) coordination polymers with circularly polarized luminescence. Chem. Commun. 54, 10695 (2018).

27. Zhang, W. Y. et al. Dramatic impact of the lattice solvent on the dynamic magnetic relaxation of dinuclear dysprosium single-molecule magnets. Inorg. Chem. Front. 5, 1575 (2018)

28. Binnemans, K. Interpretation of europium(III) spectra. Coord. Chem. Rev. 295, 1 (2015).

\section{Acknowledgements}

We are particularly grateful for experimental assistance by Professor H. Ito and Assistant Professor T. Seki of Hokkaido University. This work was supported by grant-in-aid for grant numbers 20H02748, 19H04556, 18H04497, 18H02041, 20H04653, and 20H05197.
This work was also supported by the Institute for Chemical Reaction Design and Discovery (ICReDD), established by the World Premier International Research Initiative (WPI) of MEXT, Japan. This study was supported in part by grants-in-aids for regional R\&D Proposal-Based Program from Northern Advancement Center for Science \&

Technology of Hokkaido, Japan. S.W. was also supported by The Ministry of Education, Culture, Sports Science and Technology through Program for Leading Graduate Schools (Hokkaido University 'Ambitious Leader’s Program').

\section{Author contributions}

Y.K. designed research and performed photophysical measurements of $\mathrm{Eu}$ (III) complexes. S.W. found the Eu-lumino glass with large CPL property. S.W. and M.D.J.I. performed synthesis of $\mathrm{Eu}(\mathrm{III})$ complexes and their measurements. M.G. and K.T. prepared spin-coat films and support CPL measurements. K.S. and S.M. calculated the structure of $\mathrm{Eu}(\mathrm{III})$ complex. Y.K., S.W., K.F., and Y.H. wrote the paper. All authors reviewed the manuscript.

\section{Competing interests}

The authors declare no competing interests.

\section{Additional information}

Supplementary information is available for this paper at https://doi.org/10.1038/s42004 020-00366-1.

Correspondence and requests for materials should be addressed to Y.K. or Y.H.

Reprints and permission information is available at http://www.nature.com/reprints

Publisher's note Springer Nature remains neutral with regard to jurisdictional claims in published maps and institutional affiliations.

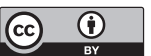

Open Access This article is licensed under a Creative Commons Attribution 4.0 International License, which permits use, sharing, adaptation, distribution and reproduction in any medium or format, as long as you give appropriate credit to the original author(s) and the source, provide a link to the Creative Commons license, and indicate if changes were made. The images or other third party material in this article are included in the article's Creative Commons license, unless indicated otherwise in a credit line to the material. If material is not included in the article's Creative Commons license and your intended use is not permitted by statutory regulation or exceeds the permitted use, you will need to obtain permission directly from the copyright holder. To view a copy of this license, visit http://creativecommons.org/ licenses/by/4.0/

(c) The Author(s) 2020 